\title{
Summaries and abstracts
}

Climate policy after Paris: assessment and perspectives, by Marinella Davide, Elisa Calliari and Aurora D'aprile

The Paris Agreement, adopted at the end of 2015 has been welcomed as a turning point in climate negotiations, after the failed attempt at Copenhagen climate conference in 2009 and four years of groundwork started in 2011 in Durban. Its mandate was indeed to adopt "a protocol, another legal instrument or an agreed outcome with legal force" applicable to all the States Parties to the UNFCCC. The Paris Conference (COP21) accomplished this task by delivering a global agreement that asks the international community to keep the increase in the global average temperature to well below $2{ }^{\circ} \mathrm{C}$ above pre-industrial levels, but also include adaptation and low-carbon finance objectives. To achieve these goals the Agreement formalizes a new approach, applicable to all countries, and that will rely on "Nationally Determined Contributions". Yet, beyond the text, challenges remain for turning commitments into action. Key technical and political elements are still to be detailed in policy areas such as mitigation, adaptation, carbon pricing, climate finance and transparency. This paper aims at examining the main features of the new global climate agreement, paying particular attention to these key issues and discussing the implications they will have in the near and long term future.

Keywords: Paris Agreement, NDCs, mitigation, adaptation, carbon pricing, climate finance.

JEL classification: K33, Q54

Sustainable development as an opportunity for innovation in the management model of public organisations, by José Aureliano Martín-Segura, José Luis Navarro-Espigares, César Pérez-López and Guillermo Maraver-Tarifa

The literature recognises the great influence that environmental spending exerts on implementing LA21. But some authors raise doubts about the firm commitment of local government. Thus the main objective of this paper is to verify the authenticity of political commitment towards meeting the objectives of sustainable development by means of the budgetary support to these policies. To accomplish this verification, we apply the Difference in Differences technique for the period 2002-2012 in the Spanish municipalities. Results obtained confirmed the initial hypothesis and show that local governments that adhered to LA21 increased their environmental expenses to a greater degree.

Keywords: Local Agenda 21, environmental expenses, difference in differences.

JEL classification: H76, H72, Q58, C21, C33

Economics and policy of energy and the environment, $n$. 3/2015 
Energy consumption and growth: a review of international empirical literature, by Yvonne Gwenhure and Nicholas M Odhiambo

The relationship between energy consumption and economic growth has been extensively examined, using different methodologies, in a wide range of countries across the world. This paper focuses on a review of existing literature on the causal relationship between energy consumption and economic growth. There are currently four views regarding the direction of causality between energy consumption and economic growth in the literature. The first view centres on the notion that energy consumption Granger-causes economic growth. This view is noted as the "energy-led growth hypothesis". The second view, known as the "growth-led energy consumption hypothesis", supports the view that economic growth Granger-causes energy consumption. The third view argues for a bidirectional causal relationship between energy consumption and economic growth, whilst the fourth view argues for no causal relationship between the two variables. Although other literature surveys have focused mainly on studies that use total energy consumption as a proxy for energy consumption, few have consolidated the literature based on different energy sources and how this impacts on the direction of causality. The aim of this paper is therefore to review literature on the causal relationship between energy consumption and economic growth by energy source. The findings from the literature reviewed in this study reveal that although there is no consensus yet on the direction of causality between energy consumption and economic growth, the conventional wisdom is in favour of the feedback hypothesis, where energy consumption and economic growth Granger-cause one another. In terms of energy sources, the study reveals that electricity energy consumption predominates the growth-led energy hypothesis, whilst renewable energy consumption predominates the feedback hypothesis.

Keywords: energy consumption, economic growth.

JEL classification: Q43, Q40, O13.

Comparative life cycle assessment of four insulating boards made with natural and recycled materials, by Benedetta Nucci and Fabio Iraldo

Purpose. This paper quantifies and compares the environmental impacts calculated with Life Cycle Assessment (LCA) for four different insulating boards made with natural or recovered fibers: recycled PET, recycled textile, hemp and sheep wool fibers.

Methods. The environmental impacts of the different boards are compared using as functional unit $1 \mathrm{R}$ of thermic resistance and the system boundaries are taken from cradle to grave. Moreover, the length of the use phase necessary to balance the $\mathrm{CO}_{2}$ emissions linked to the life cycle of each insulating board has been assessed.

Results and discussion. The results of the comparative LCA and a contribution analysis are reported in order to highlight the main sources of environmental impact. The insulating boards made with sheep wool and recycled textile show the lowest environmental impact for almost all the impact categories considered in the analysis and require the shortest use time in order to balance the $\mathrm{CO}_{2}$ emissions of the life cycle (5 months). If the $\mathrm{CO}_{2}$ uptake from the atmosphere is included in the calculation, the insulating board made with hemp outperform the other boards in impact category "climate change" and in the "offsetting period" (less than 1 month).

Conclusions. The paper concludes that the use of insulating boards, in particular made with recycled or natural materials, can represent an important step towards the increase of the sustainability of the building sector. In addition, LCA results should increasingly be included in rating systems and schemes in the building sector in order to effectively lead this sector toward sustainability. 
Keywords: life cycle assessment, insulation, recycled PET, sheep wool, hemp, recycled textile.

JEL classification: Q51, L74, M40.

Free municipal waste trade as an incentive to recycling. A theoretical study, by Antonio Massarutto and Francesco Silvestri

According to the Self-Sufficiency and Proximity Principle (SSP), municipal waste (MW) disposal has to be confined within delimited territorial zones, with no trade allowed. The justification is usually the need to make each community aware of its environmental responsibility and discourage diversion of MW to "waste havens". In this paper, we explore the possible benefits associated with some opening to MW treatment service trade. We assume that some areas are equipped with MW treatment facilities, while others are not. We demonstrate, under standard assumptions, that removing SSP and allowing trade of MW disposal services between such regions may foster MW selection in both areas. This is true not only when disposal capacity is constrained, so that a part of the original demand for MW diversion is unsatisfied, but also when it is unconstrained and can accommodate all the residual unsorted MW, provided that the design of rewarding schemes is appropriate. On the contrary, if both districts were equipped with a facility, the same would not be true, and each one would be more willing to exploit the plant's capacity before engaging in efforts to reduce waste.

Keywords: L43, L99, Q53, R11

JEL classification: solid waste, waste management, disposal facilities, waste trade, regulated markets. 